\title{
Preparation of folic acid-conjugated, doxorubicin- loaded, magnetic bovine serum albumin nanospheres and their antitumor effects in vitro and in vivo
}

This article was published in the following Dove Press journal:

International Journal of Nanomedicine

4 September 2014

Number of times this article has been viewed

\section{Rui Yang' \\ YanLi An ${ }^{2}$ \\ FengQin Miao' \\ MengFei $\mathrm{Li}^{\prime}$ \\ PeiDang Liu' \\ QiuSha Tang'}

'School of Medicine, Southeast University, ${ }^{2}$ Jiangsu Key Laboratory of Molecular and Functional Imaging, Department of Radiology, Zhongda

Hospital, Nanjing, Jiangsu Province,

People's Republic of China
Correspondence: QiuSha Tang

School of Medicine, Southeast University, Dingjiaqiao Road 87, Nanjing 210009 , Jiangsu Province, People's Republic of China

Tel +86 25 83272373

Fax +86 258327254 I

Email panyixi-tqs@163.com
Background: This study aimed to generate targeted folic acid-conjugated, doxorubicin-loaded, magnetic iron oxide bovine serum albumin nanospheres (FA-DOX-BSA MNPs) that lower the side effects and improve the therapeutic effect of antitumor drugs when combined with hyperthermia and targeting therapy. A new nanodrug using magnetic nanospheres for heating and addition of the folate receptor with cancer cell specificity was prepared. The characteristics of these nanospheres and their antitumor effects in nasopharyngeal carcinoma were explored.

Methods: FA-DOX-BSA MNPs comprising encapsulated magnetic iron oxide nanoparticles were prepared using a desolvation cross-linking method. Activated folic acid (Nhydroxysuccinimide ester of folic acid) was conjugated to the surface of albumin nanospheres via amino groups.

Results: Folic acid was successfully expressed on the surface of the nanospheres. Electron microscopy revealed that the FA-DOX-BSA MNPs were nearly spherical and uniform in size, with an average diameter of $180 \mathrm{~nm}$. The nanomaterial could deliver doxorubicin at clinically relevant doses with an entrapment efficiency of $80 \%$. An increasing temperature test revealed that incorporation of magnetic iron oxide into nanospheres could achieve a satisfactory heat treatment temperature at a significantly lower dose when placed in a high-frequency alternating magnetic field. FA-DOX-BSA MNPs showed greater inhibition of tumors than in the absence of folic acid in vitro and in vivo. Compared with chemotherapy alone, hyperthermia combined with chemotherapy was more effective against tumor cells.

Conclusion: Folic acid-conjugated bovine serum albumin nanospheres composed of mixed doxorubicin and magnetic iron oxide cores can enable controlled and targeted delivery of anticancer drugs and may offer a promising alternative to targeted doxorubicin therapy for nasopharyngeal carcinoma.

Keywords: doxorubicin, bovine serum albumin, folic acid, KB cells

\section{Introduction}

Nasopharyngeal carcinoma (NPC) is a common cancer and has a relatively high incidence in southern China, Southeast Asia, and North Africa. ${ }^{1}$ Annually, approximately 80,000 new cases of oral cancer are reported worldwide. Death will occur in more than $60 \%$ of these cases. ${ }^{2}$ NPC is an Epstein-Barr virus-related cancer, in which radiation and chemotherapy demonstrate antitumor effects but cause injury to normal tissue cells to varying degrees, leading to a poor prognosis and high mortality after treatment. Despite improvement in the clinical cure rate for early-stage NPC, multidrug resistance and side effects encountered in advanced or intracranially recurrent NPC remain issues to be addressed. Thus, identifying more aggressive treatment strategies for NPC would be beneficial. 
Chemotherapy has long been used in the treatment of many cancers. Doxorubicin, an anthracycline antibiotic, is an effective chemotherapeutic agent in numerous types of tumor, including solid malignancies, acute leukemias, and malignant lymphoma. ${ }^{3}$ However, its use has been limited by concomitant dose-related and irreversible cardiotoxicity, as well as the emergence of drug resistance. ${ }^{4}$ Damage to nontargeted tissues often stymies cancer therapy by limiting the doxorubicin dosage. ${ }^{5}$ The underlying causes of drug resistance remain unknown, so it is important to establish delivery systems to minimize doxorubicin-induced toxicity while obtaining maximum anticancer efficacy. The framework used in drug nanocarrier systems comprises mainly two materials, ie, natural and synthetic polymers. Natural polymers, particularly albumin with its unique physical and chemical attributes, are versatile protein carriers for drug targeting and improving the pharmacokinetic profiles of peptide-based or protein-based drugs. ${ }^{6}$ The particle size of serum albumin is at the nanoscale to mesoscale level. Additionally, serum albumin is a polymer consisting of amino acids linked by peptide bonds, which facilitates the embedding and loading of drugs. Carriage of drugs by serum albumin improves their biodegradability and stability, and enables slow release and uptake by the tumor and inflamed tissue. ${ }^{7}$ Furthermore, its numerous lysine residues facilitate chemical coupling and modification of bovine serum albumin. These characteristics suggest that this protein can transport therapeutic drugs. Therefore, we prepared doxorubicin-loaded serum albumin nanoparticles to enhance the antitumor effects by using serum albumin as drug carrier.

Nanotechnology is attracting great attention worldwide in biomedicine. Targeted therapy based on drug nanocarrier systems enhances the treatment of tumors and enables the development of targeted drug delivery systems. Compared with nontargeted drug delivery systems, targeted systems not only increase therapeutic efficacy and reduce the side effects of antitumor drugs but also enhance targeting and tissue absorption. Receptor-ligand interactions represent the most important method of targeting antitumor drugs to living cells. The focus has been on identifying highly specific receptors in the membranes of tumor cells that demonstrate a significantly different drug uptake ability compared with nontarget tissues. The folic receptor, also known as folic acid-binding protein, is expressed at a low level in normal cells, but is overexpressed during cellular activation and proliferation, particularly in human tumors such as NPC, ovarian cancer, and gliomas. Consequently, the folic receptor is a promising target ligand for cancer treatment. Folic acid is essential for the maintenance of cell functions. Binding of folic acid to the folic receptor results in receptor-mediated endocytosis and internalization. The functionalized polylactide-co-glycolidepolyethylene glycol-folic acid nanoparticles containing anticancer drugs developed by Saxena et al showed a two-fold increase in cytotoxicity and considerably higher intracellular uptake of folic-targeted nanoparticles compared with free drug or nontargeted nanoparticles in MCF-7 human breast cancer cells. ${ }^{8}$ Further, Lee et al prepared folic acid/secondary lymphoid tissue chemokine (CCL21)/upconversion fluorescent nanoparticle conjugates that can target folic receptorexpressing tumor cells and successfully attract immune cells. ${ }^{9}$ Other ligands, such as antibodies and hormones, are transported to lysosomes and cleared. ${ }^{10,11}$ Together, the above data suggest that folic acid may be a good ligand for targeting of tumors.

Heat treatment is an ancient and promising method for treating tumors and was first used in 5,000 BC by the ancient Egyptians to treat breast cancer. ${ }^{12}$ Heating tissues to $41^{\circ} \mathrm{C}-45^{\circ} \mathrm{C}$ to achieve the therapeutic aim was known as green therapy. The combination of chemotherapy or radiotherapy with heat not only enables a decreased dose of chemotherapy or radiotherapy to be applied, thus reducing side effects, but also can improve therapeutic efficacy by enhancing the killing of tumor cells. Pelicci et al evaluated the antitumor effect of heat in combination with radiotherapy. The combination of increased temperature at the tumor site generated by laser treatment of intravenously injected gold nanoshells and ionizing radiation enhanced both the radiosensitivity of cancer stem cells and the tumor response. The results suggested that heating is a potent radiation sensitizer if provided appropriately. ${ }^{13}$ The drawbacks of heat treatment include the possibility of failure to deliver and monitor the temperature in deep-seated tumors or use of an inefficient traditional-type heater that has long been out of use. Magnetic-targeted heat treatment, initially proposed by Gilchrist et al has promoted heat treatment for tumors because it can precisely deliver heat to the target. ${ }^{14}$ The benefits of using magnetic nanomaterials for tumor heating are that they can enter tumor tissue either alone or as conjugates with small molecules, or be included in bulky cargos such as liposomes or proteins. ${ }^{15-17}$ The temperature of the tissue can be effectively controlled by an external magnetic field; therefore, no thermal damage to nontarget zones occurs. Magnetic nanoparticles, particularly magnetic fluids, are a new heating technology for the treatment of tumors and were first reported by Jordan et al in 1997. ${ }^{18}$ Magnetic fluids are nanostructured materials with magnetic properties. Iron oxide $\left(\mathrm{Fe}_{3} \mathrm{O}_{4}\right)$ displays considerable magnetism, catalysis, and wave absorption properties, making it the most commonly 
used magnetic fluid for tumor hyperthermia. ${ }^{19,20}$ However, $\mathrm{Fe}_{3} \mathrm{O}_{4}$ has a short half-life and no specific tumor-targeting effect because it is readily taken up by reticuloendothelial cells and removed by macrophages. We have designed bovine serum albumin nanospheres containing folic acid-conjugated $\mathrm{Fe}_{3} \mathrm{O}_{4}$ to improve the pharmacokinetic profile and targeting effect, due to the long half-life of albumin in the body and binding of folic acid to the folic receptor.

In the present study, we used bovine serum albumin nanospheres containing $\mathrm{Fe}_{3} \mathrm{O}_{4}$ and doxorubicin conjugated to folic acid (FA-DOX-BSA MNPs). We compared the ability of various FA-DOX-BSA MNPs to inhibit tumors, including doxorubicin-loaded bovine serum albumin nanospheres (DOX-BSA NPs), magnetic bovine serum albumin nanospheres (BSA MNPs), doxorubicin-loaded magnetic bovine serum albumin nanospheres (DOX-BSA MNPs), and FA-DOX-BSA MNPs in vivo and in vitro, to investigate whether targeted delivery is effective for NPC therapy. The tumor-targeting efficacy of FA-DOX-BSA MNPs in vitro and in vivo has been confirmed by our laboratory. ${ }^{21}$

\section{Materials and methods Materials}

Bovine serum albumin, 3-(4,5-dimethyl-2-thiazyl)-2, 5-diphenyl-2H-tetrazolium bromide (MTT), dimethyl sulfoxide, phosphate-buffered saline ( $\mathrm{pH} 7.4)$, N-dicyclohexylcarbodiimide, dicyclohexylurea, N-hydroxysuccinimide (NHS), and doxorubicin hydrochloride were purchased from Sigma-Aldrich (St Louis, MO, USA). Ammonium ferric sulfate dodecahydrate, hydroxylamine hydrochloride, 1,10phenanthroline, and sodium acetate were purchased from JiangLai Biological (Shanghai, People's Republic of China). All solvents were of high-performance liquid chromatography grade. An Annexin V-fluorescein isothiocyanate (FITC) apoptosis detection kit was supplied by Beyotime Institute of Biotechnology (Shanghai, People's Republic of China). $\mathrm{Fe}_{3} \mathrm{O}_{4}$ was prepared as described by $\mathrm{Li}$ et al. ${ }^{22}$ All reagents were of analytical grade.

\section{Synthesis of targeting FA-DOX-BSA MNPs}

DOX-BSA MNPs were firstly prepared using a desolvation cross-linking technique. Briefly, bovine serum albumin $(0.25 \mathrm{~g}), \mathrm{Fe}_{3} \mathrm{O}_{4}(0.05 \mathrm{~g})$, and doxorubicin $(0.01 \mathrm{mg})$ were dissolved in $20 \mathrm{~mL}$ of deionized water. The $\mathrm{pH}$ was adjusted to 8.0 after thorough mixing. Absolute alcohol $(150 \mathrm{~mL})$ was added to the solution, followed by stirring at room temperature for 1-2 hours until a precipitate appeared. Next, $0.25 \%$ glutaraldehyde $(50 \mu \mathrm{L})$ solution was added to cross-link the amino groups in the nanoparticles. This process was performed during stirring for 12 hours. Finally, the mixture was centrifuged at $1,500 \mathrm{rpm}$ for 5 minutes to remove nonencapsulated $\mathrm{Fe}_{3} \mathrm{O}_{4}$, and then at 21,000 rpm for 30 minutes to remove nonencapsulated doxorubicin, followed by washing three times with deionized water. The solution was redispersed in $3.0 \mathrm{~mL}$ of purified water.

The NHS ester of folic acid (folic-NHS) was prepared as follows. Folic acid (0.5 g) was dissolved in dimethyl sulfoxide $(10 \mathrm{~mL})$, to which was added triethylamine $(0.25 \mathrm{~mL})$. N-dicyclohexylcarbodiimide (4.8 g) and NHS (2.6 g) were added to the folic acid-dimethyl sulfoxide solution while stirring. The reaction was continued at room temperature overnight. Dicyclohexyl urea, which is the byproduct of the synthesis of folic-NHS, was removed by filtering. After these steps, we obtained folic-NHS

FA-DOX-BSA MNPs was prepared by conjugating DOX-BSA MNPs with folic acid via amino groups. $\mathrm{NaCO}_{3} /$ $\mathrm{NaHCO}_{3}$ buffer ( $\mathrm{pH}$ 11.5) was added to a solution of DOXBSA MNPs $(3 \mathrm{~mL})$ to adjust the $\mathrm{pH}$ to 10.0 . The dimethyl sulfoxide solution of folic-NHS (1 mL) was added to the solution, followed by stirring at room temperature for 2 hours. The reactants were placed in a dialysis bag $(3,500 \mathrm{Da})$ to remove the dimethyl sulfoxide. A Sephadex G-50 column was used to separate the FA-DOX-BSA MNPs from unreacted folic acid and other byproducts. The eluent containing FA-DOX-BSA MNPs was centrifuged at 21,000 rpm for 30 minutes and the pellets were redispersed in $4.0 \mathrm{~mL}$ of purified water.

\section{Characterization of targeted FA-DOX-BSA MNPs}

Assessment of morphology by transmission electron microscopy

The FA-DOX-BSA MNP sample was redispersed in an ultrasonication bath for 15 minutes and then dripped onto a copper net with carbon support film to prepare for visualization using a transmission electron microscope (Hitachi, Tokyo, Japan).

\section{Dynamic light scattering analysis}

The particle size and size distribution of the FA-DOXBSA MNPs were assessed by dynamic light scattering (Brookhaven Instruments Co, Holtsville, NY, USA). To assess the stability of the nanoparticles, the hydrodynamic size of the nanoparticles in phosphate-buffered saline or Roswell Park Memorial Institute (RPMI) 1640 medium supplemented with $10 \%$ fetal bovine serum was analyzed by dynamic light scattering within 24 hours. Particle size was measured for 18 days with storage at $4^{\circ} \mathrm{C}$ and ambient humidity. 


\section{Iron content, specific absorption rate,}

\section{and increasing temperature tests}

Iron content was measured using 1,10-phenanthroline spectrophotometry based on the principle that 1,10-phenanthroline generates stable color complexes in aqueous solution at pH 2-9. The steps were as follows. First, $5 \mu \mathrm{L}$ of FA-DOXBSA MNP solution was brought up to $1 \mathrm{~mL}$ with $1 \mathrm{M}$ $\mathrm{HCl}$ and blended in an ultrasonication bath. Second, 10\% hydroxylamine hydrochloride $(1 \mathrm{~mL}), 0.1 \%$ phenanthroline solution $(2 \mathrm{~mL})$, acetic acid, and sodium acetate buffer (pH 5, $5 \mathrm{~mL}$ ) were added, and the solution was brought up to $50 \mathrm{~mL}$ with ultrapure water. A standard solution was prepared by dissolving an accurately measured quantity of $\mathrm{FeCl}_{3} \cdot 6 \mathrm{H}_{2} \mathrm{O}$ according to the second step. The different optical density (OD) values were detected by ultraviolet spectrophotometry (DU 800, Beckman Coulter, Brea, CA, USA) at $510 \mathrm{~nm}$. A standard curve was constructed using the iron content of the standard solution $(\mathrm{mg})$ as the abscissa and the OD value as the $y$ coordinate. The regression equation of the iron content and the OD value was also derivative. The iron concentration in the sample solution was determined from the standard regression equations. The sample was diluted proportionally in ultrapure water to the appropriate concentrations. Next, the solution was placed in a flat-bottomed test tube on an SPG-06A high-frequency induction heater from Shenzhen, People's Republic of China ( $\mathrm{f}=200 \mathrm{kHz}$; I $=20 \mathrm{~A}$ ) for 60 minutes. The distance between the bottom of the tube and center of the heating coil of the alternating magnetic field was $0.5 \mathrm{~cm}$. The temperature was tested using a digital thermometer at 5-minute intervals. The specific absorption rate (SAR) values for $\mathrm{Fe}_{3} \mathrm{O}_{4}$ and $\mathrm{FA}-\mathrm{DOX} / \mathrm{BSA}$ MNPs were calculated using the following formula:

$$
\mathrm{SAR}=C \frac{d T}{d t} \frac{m_{s}}{m_{m}},
$$

where $C$ is the specific heat capacity of the suspension, $d T / d t$ is the initial slope of temperature versus time graph, $m_{s}$ is the mass of the suspension, and $m_{m}$ is the mass of the magnetic material in the suspension.

\section{Drug encapsulation, loading, and cumulative drug release}

Drug encapsulation efficiency was measured using an ultraviolet-visible near-infrared spectrophotometer (UV-3600; Shimadzu, Tokyo, Japan). First, $1 \mathrm{~mL}$ of FA-DOX-BSA MNPs was dispersed into $9 \mathrm{~mL}$ of $0.5 \%$ pepsin aqueous solution and digested for 3 hours at $37^{\circ} \mathrm{C}$. The supernatant was collected after centrifuging for 30 minutes at $21,000 \mathrm{rpm}$ and detected at $480 \mathrm{~nm}$ using ultraviolet spectrophotometry. Drug encapsulation efficiency was calculated as follows: (actual amount of drug encapsulated in MNPs)/(initial amount of drug used in the fabrication of MNPs) $\times 100 \%$. Drug loading was calculated as follows: (actual amount of drug encapsulated in MNPs)/(amount of MNPs) $\times 100 \%$. Doxorubicin release from the nanoparticles in phosphate-buffered saline with three different $\mathrm{pH}$ values ( $\mathrm{pH} 5.4,7.4$, and 9.0) at $37^{\circ} \mathrm{C}$ was evaluated using the dynamic dialysis method. A sample $(15 \mathrm{~mL})$ was placed in a dialysis bag (molecular weight cutoff $3,500 \mathrm{Da}$ ), which was then immersed in a $50 \mathrm{~mL}$ centrifuge tube containing $30 \mathrm{~mL}$ of phosphate-buffered saline. The tube was placed in a horizontal shaking incubator (Shanghai, People's Republic of China) at $37^{\circ} \mathrm{C}$ and $110 \mathrm{rpm}$. A $5 \mathrm{~mL}$ sample was removed from the centrifuge tube and replaced with $5 \mathrm{~mL}$ of fresh buffer at regular time intervals. Doxorubicin release from the nanoparticles in phosphate-buffered saline ( $\mathrm{pH} 7.4$ ) at $43^{\circ} \mathrm{C}$ was also evaluated using the same methods. The doxorubicin concentration in each sample was quantified by ultraviolet spectrophotometry at $480 \mathrm{~nm}$. The cumulative release rate, Q (\%), was calculated as follows:

$$
Q(\%)=\sum_{i=1}^{n=j} \mathrm{CiVi}+\mathrm{CnV} / W \times D \times 100 \%
$$

where $\mathrm{Ci}$ and $\mathrm{Cn}$ are the drug concentration in released mediators, $V i$ is the volume of mediator removed, $V$ is the total amount of leaching mediator, and $W$ and $D$ are the weight of the microspheres and drug concentration in the microspheres, respectively.

\section{Surface functional groups}

An ultraviolet-visible near-infrared spectrophotometer was used to determine whether folic acid was conjugated to the DOX-BSA MNPs.

\section{Toxicity assessment}

As we know, folic acid is essential for the maintenance of cell functions, bovine serum albumin has good biodegradability, and both are nontoxic to normal cells. Our laboratory has been studying the applications of $\mathrm{Fe}_{3} \mathrm{O}_{4}$ for a long time. It shows good biocompatibility, which has been proven by Chen et al. ${ }^{23}$ So the folic acid conjugated MNPs also have good biodegradability.

\section{Antitumor effects of FA-DOX-BSA MNPs in vitro}

Cell culture

NPC KB cells were obtained from the Shanghai Institute of Cell Biology (Shanghai, People's Republic of China) and 
cultured in folic acid-free RPMI 1640 medium (Gibco, Grand Island, NY, USA) supplemented with $10 \%$ fetal bovine serum (Shanghai, People's Republic of China), 100 U/mL penicillin, and $100 \mathrm{mg} / \mathrm{mL}$ streptomycin. Cells were maintained at $37^{\circ} \mathrm{C}$ with $5 \% \mathrm{CO}_{2}$ in a humidified incubator.

\section{MTT}

The antitumor effect of FA-DOX-BSA MNPs on KB cells was measured by MTT assay. KB cells were seeded in 96-well tissue culture plates at a density of $5 \times 10^{4}$ per well and cultured in folic acid-free RPMI 1640 medium for 24 hours. Subsequently, the cells were divided into the following groups: (1) a negative control group (folic acid-free RPMI 1640 medium containing 10\% fetal calf serum); (2) a DOX-BSA NP group; (3) a DOX-BSA MNP group with no heating; (4) a FA-DOX-BSA MNP group with no heating; (5) a BSA MNP group with heating; (6) a DOX-BSA MNP group with heating; (7) a FA-DOX-BSA MNP group with heating; (8) a negative control group with heating (folic acidfree RPMI 1640 medium containing 10\% fetal calf serum); and (9) a BSA MNP group with no heating. Groups 5, 6, 7, and 8 were placed on the heating coil of a high-frequency induction heater $(\mathrm{f}=200 \mathrm{kHz}$; $\mathrm{I}=20 \mathrm{~A})$ for 60 minutes. After incubation for 48 hours, MTT solution $(20 \mu \mathrm{L})$ was added to all groups, followed by incubation at $37^{\circ} \mathrm{C}$ for 4 hours. Next, dimethyl sulfoxide $(200 \mu \mathrm{L})$ was added, and the solution was mixed until the precipitate dissolved. The 96-well plate was read at $590 \mathrm{~nm}$ using a spectrophotometer (Bio-Tek, Winooski, VT, USA). The cell growth inhibition rate (IR) was calculated using the following formula: $\mathrm{IR}=(1-$ the experimental group OD/the negative control group OD) $\times 100 \%$.

\section{Apoptosis assay}

The apoptosis rate was determined by flow cytometry. The KB cells were divided into nine groups and treated as described above. The cells were then collected into tubes and washed with phosphate-buffered saline. Annexin V-FITC $(5 \mu \mathrm{L})$ and propidium iodide $(5 \mu \mathrm{L})$ were added to the tubes, followed by incubation at $25^{\circ} \mathrm{C}$ for 10 minutes in the dark. The effect of FA-DOX-BSA MNPs on the apoptosis rate was evaluated using flow cytometry (FACSCalibur; Becton Dickinson, Mountain View, CA, USA). All experiments were performed in triplicate.

\section{Antitumor effect of FA-DOX-BSA MNPs in vivo}

$\mathrm{BALB} / \mathrm{C}$ nude mice (male, aged 5 weeks) were purchased from the Shanghai Institute of Biological Sciences, Chinese Academy of Sciences (Shanghai, People's Republic of
China). Xenograft tumors were induced by subcutaneous injection of $\mathrm{KB}$ cells under the right leg. After 2 weeks, the mice were divided into five groups of six mice each as follows: a negative control group (folic acid-free RPMI medium containing $10 \%$ fetal calf serum); a DOX-BSA NP group; a BSA MNP group with heating; a FA-DOX-BSA MNP group with no heating; and a FA-DOX-BSA MNP group with heating. The mice had been injected intraperitoneally with sodium pentobarbital $(50 \mathrm{mg} / \mathrm{kg})$ beforehand. Next, all solutions were directly injected into the tumors at the 3,6, 9, and 12 o'clock positions, at a volume equal to half of the tumor volume. After 24 hours, all of the groups were placed on a high-frequency induction heater ( $f=200 \mathrm{kHz} ; \mathrm{I}=20 \mathrm{~A}$ ) for 60 minutes every other day. The maximal temperature of the rectal tissue did not exceed $40^{\circ} \mathrm{C}$, and the maximal temperature of the tumor core was maintained at $50^{\circ} \mathrm{C}$. The tumor volume was calculated as follows: (long diameter $\times$ short diameter ${ }^{2}$ ) $/ 2$. The rate of inhibition of tumor volume was calculated as (1- volume of experimental group/volume of control groups) $\times 100 \%$. Six days later, all of the mice were euthanized, and then weighed and sectioned with hematoxylin-eosin staining. The rate of inhibition of tumor weight was calculated as follows: (1-weight of experimental group/weight of control groups) $\times 100 \%$.

\section{Statistical analysis}

Values are shown as the mean \pm standard deviation. The data were analyzed with Statistical Package for the Social Sciences version 16.0 software (SPSS Inc., Chicago, IL, USA). A $P$-value of $<0.05$ was considered to be statistically significant.

\section{Results and discussion Characterization of targeted FA-DOX-BSA MNPs}

Assessment of morphology by transmission electron microscopy

DOX-BSA MNPs was prepared using a desolvation crosslinking method with ethanol as the desolvating agent and $0.25 \%$ glutaraldehyde as the cross-linking agent. During the desolvation process, the $\mathrm{pH}$ was adjusted to 8.0 after bovine serum albumin, $\mathrm{Fe}_{3} \mathrm{O}_{4}$, and doxorubicin were dissolved in deionized water because $\mathrm{pH}$ is important for maintenance of the structure and characteristics of albumin, which has the ability to undergo a major reversible conformational isomerization with changes in $\mathrm{pH}$. Foster classified the $\mathrm{pH}-$ dependent forms of albumin as N, B, F, E, and A. ${ }^{24,25}$ When 


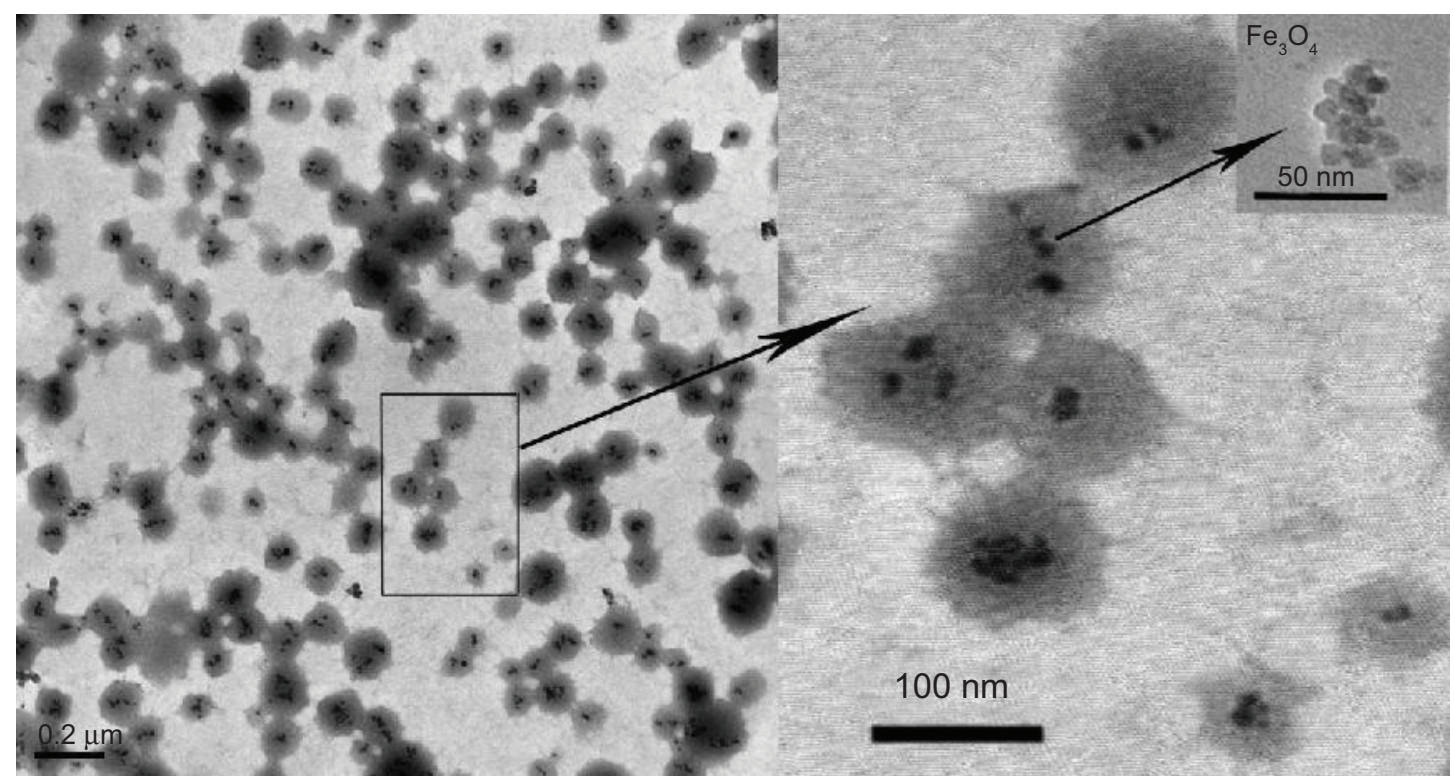

Figure I Morphology of nanoparticles by transmission electron microscopy.

Note: The particles were approximately round in shape and their size was about $100 \mathrm{~nm}$ in diameter. $\mathrm{Fe}_{3} \mathrm{O}_{4}$ could be seen in the particles with a hydrodynamic size of $20 \mathrm{~nm}$.

$\mathrm{pH} \geq 8$, the structure of albumin is $\mathrm{F}$ and the albumin molecule expands by dissociation of the $\mathrm{C}$-terminal half. Albumin at $\mathrm{F}$ has an increased viscosity and decreased dissolvability, ${ }^{26}$ which aids its formation into nanospheres. Transmission electron microscopy (Figure 1) showed FA-DOX-BSA MNPs to be approximately round in shape, and this has also been demonstrated by $\mathrm{Ak}$ et $\mathrm{al}^{27}$ and Shen et al. ${ }^{28}$ The particles were uniform in size. Moreover, an $\mathrm{Fe}_{3} \mathrm{O}_{4}$ area in the particle center could be seen in all particles. Its hydrodynamic size was about $20 \mathrm{~nm}$.

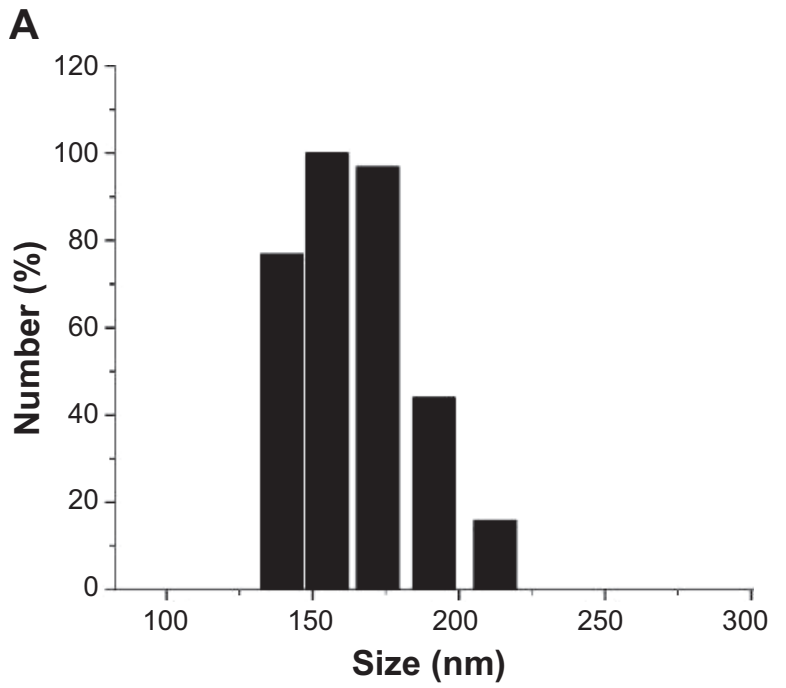

\section{Dynamic light scattering analysis}

Nanoparticle size and size distribution as measured by dynamic light scattering is shown in Figure 2A. The size of FA-DOX-BSA MNPs was $180 \pm 2.2 \mathrm{~nm}$. To assess the stability of the nanoparticles, the hydrodynamic size of the FA-DOX-BSA MNPs was analyzed by dynamic light scattering. The hydrodynamic sizes did not change significantly over 24 hours in phosphate-buffered saline or RPMI 1640 medium supplemented with $10 \%$ fetal bovine serum (Figure 2B). In addition, the nanoparticles showed limited

B

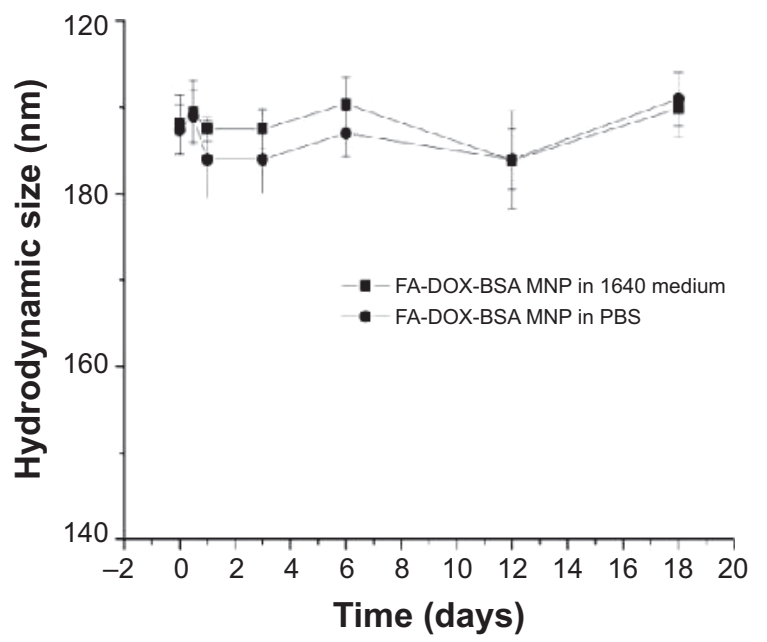

Figure 2 (A) Analysis of nanoparticle size and size distribution by dynamic light scattering. The main size distribution is I50 nm-I72 nm. (B) Hydrodynamic size of FA-DOXBSA MNP by dynamic light scattering in PBS or RPMI 1640 medium supplemented with $10 \%$ fetal bovine serum.

Abbreviations: FA, folic acid; DOX, doxorubicin; BSA, bovine serum albumin; MNPs, magnetic nanoparticles; PBS, phosphate-buffered saline; RPMI, Roswell Park Memorial Institute. 
$(<10 \%)$ variation in hydrodynamic size after 3 weeks of storage in phosphate-buffered saline at $4^{\circ} \mathrm{C}$, indicating excellent stability in aqueous medium.

\section{Iron content, SAR and increasing-temperature tests}

When the iron concentration was $0-100 \mu \mathrm{g} / \mathrm{mL}$, the regression equation was $Y=0.00417 X+0.01099, r^{2}=0.9997$, where $X$ and $Y$ are the OD values and iron concentration, respectively. The iron content was $2.2 \mathrm{mg} / \mathrm{mL}$. The SAR value for FA-DOXBSA MNPs was $60 \pm 2.1 \mathrm{w} / \mathrm{g}$. This result is consistent with that obtained for $\mathrm{Fe}_{3} \mathrm{O}_{4}$ alone $(59.7 \pm 3.2 \mathrm{~W} / \mathrm{g})$. When the alternating magnetic field intensity was fixed, a perfect correlation between the heating ability of the FA-DOX-BSA MNPs and iron content was obtained, ie, the greater the iron concentration, the greater the increase in temperature. The heating curves for the various iron concentrations were similar: during the first 30 minutes, the temperature of the sample increased rapidly, and then only slightly between 30 and 35 minutes. After 35 minutes, the temperature remained constant (Figure 3). The $0.375 \mathrm{mg} / \mathrm{mL}$ solution could reach a temperature of $43^{\circ} \mathrm{C}$ (effective treatment temperature is $41^{\circ} \mathrm{C}-44^{\circ} \mathrm{C}$ ) and the temperature could remain stable. Therefore, $0.375 \mathrm{mg} / \mathrm{mL}$ was selected for follow-on magnetic fluid hyperthermia in our experiment.

\section{Drug encapsulation efficiency, drug loading efficiency and drug release}

We used bovine serum albumin nanospheres containing $\mathrm{Fe}_{3} \mathrm{O}_{4}$ and doxorubicin encapsulated by a direct drug-loading process. For direct drug-loading, bovine serum albumin solution was prepared and doxorubicin and $\mathrm{Fe}_{3} \mathrm{O}_{4}$ were mixed into it;

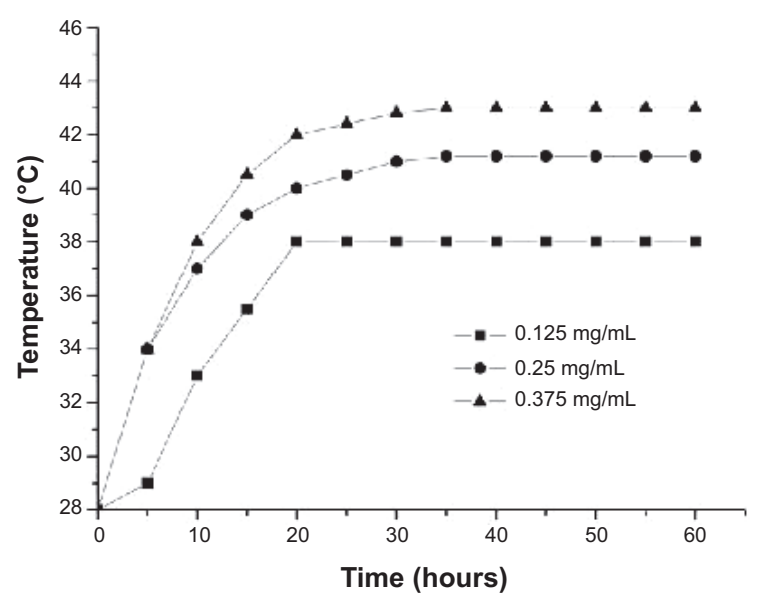

Figure 3 Heating curve for FA-DOX-BSA MNPs.

Note: When exposed to a high-frequency AMF ( $f=20 \mathrm{kHz} ; \mathrm{I}=20 \mathrm{~A})$, the nanospheres in different iron content rapidly reached the temperature required for heat treatment $\left(41^{\circ} \mathrm{C} \sim 44^{\circ} \mathrm{C}\right)$ within 30 minutes, which remained stable after 30 minutes.

Abbreviations: FA, folic acid; DOX, doxorubicin; BSA, bovine serum albumin; MNPs, magnetic nanoparticles. ethanol was then added dropwise in a continuous manner. Drug encapsulation efficiency and drug loading efficiency of nanoparticles are crucial for their clinical application. In our study, we found a favorable drug encapsulation efficiency, drug loading efficiency, and drug release time. Drug loading efficiency was $\sim 4 \%$ and drug encapsulation efficiency was $\sim 80 \%$. Release of doxorubicin from the samples was determined by ultraviolet spectrophotometry, and the cumulative release at various time points in three $\mathrm{pH}$ solutions ( $\mathrm{pH}$ 5.4, 7.4, 9.0) was calculated (Figure 4). Cumulative release of FA-DOX-BSA MNPs at 72 hours in $\mathrm{pH} 7.4$ solutions was less than $6 \%$. However, it had significantly increased in a weak acid medium, with a cumulative release rate of $78.3 \%$. The magnetic nanoparticles were very stable at neutral and alkaline $\mathrm{pH}$. No significant difference in cumulative release rate was observed in the presence of heating. The $\mathrm{pH}$ values in tumor tissues and lysosome were slightly acidic. With the magnetic nanoparticles inside the cancer cells, doxorubicin would release from it. However, when the magnetic nanoparticles in normal tissues, the doxorubicin would not release. This result confirmed that bovine serum albumin could carry anticancer drugs, which would be stable in blood during in vivo circulation and be released quickly after being selectively taken up by tumor tissue. Better release properties help chemotherapy drugs to have a stronger inhibitory and killing effect on cancer cells, which may improve patient acceptance and compliance.

\section{Surface functional groups}

Ultraviolet-visible near-infrared spectrophotometry was used to confirm the binding of folic acid to bovine serum

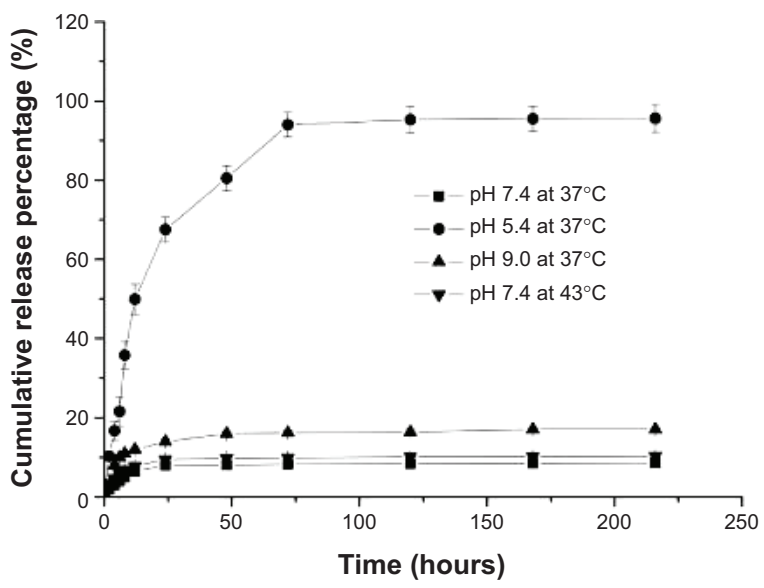

Figure 4 Cumulative in vitro profile of doxorubicin release from FA-DOX-BSA MNPs.

Note: Doxorubicin released from FA-DOX-BSA MNPs was evaluated using the dynamic dialysis method in different $\mathrm{pH}$ phosphate-buffered saline solutions $(\mathrm{pH} 5.4$, 7.4, and 9.0) at $37^{\circ} \mathrm{C}$ and in phosphate-buffered saline solutions ( $\mathrm{pH} \mathrm{7.4)}$ at $43^{\circ} \mathrm{C}$.

Abbreviations: FA, folic acid; DOX, doxorubicin; BSA, bovine serum albumin; MNPs, magnetic nanoparticles. 


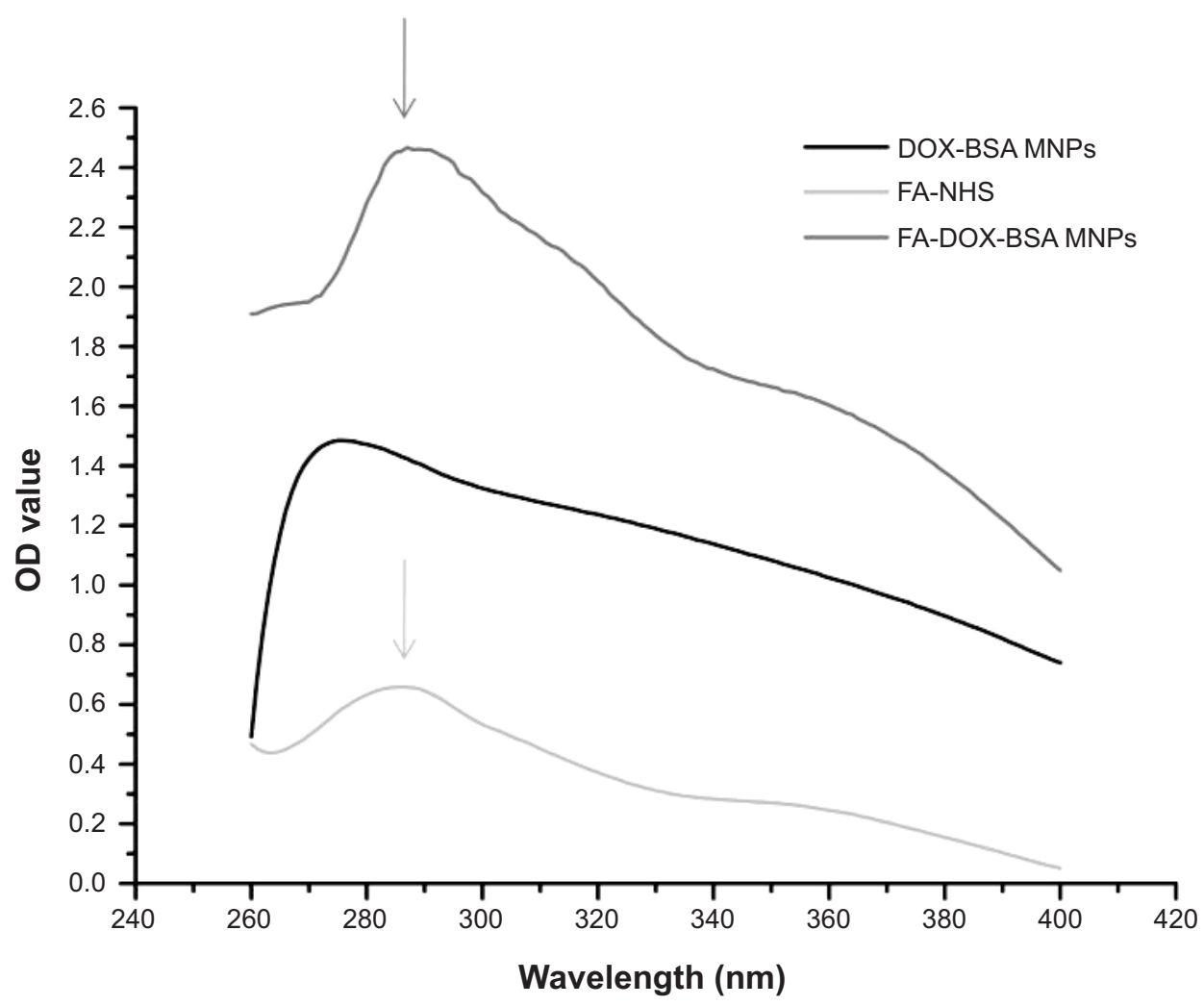

Figure 5 Ultraviolet spectra of FA-NHS, DOX-BSA MNPs, and FA-DOX-BSA MNPs.

Note: The maximum absorption wavelength of FA-NHS appeared between $280 \mathrm{~nm}$ and $300 \mathrm{~nm}$ (dark gray arrow), which was consistent with the absorption wavelength of FA-DOX-BSA MNPs (light gray arrow).

Abbreviations: OD, optical density; FA, folic acid; DOX, doxorubicin; BSA, bovine serum albumin; MNPs, magnetic nanoparticles; NHS, n-hydroxysuccinimide.

albumin. The ultraviolet spectra for FA-NHS, DOX-BSA MNPs, and FA-DOX-BSA MNPs are shown in Figure 5. An absorption maximum for FA-NHS of 250-300 nm was detected by ultraviolet-visible near-infrared spectrophotometry. The absorption peak of FA-DOX-BSA MNPs at 286 $\mathrm{nm}$ clearly indicated successful contact between folic acid and DOX-BSA MNPs.

\section{Antitumor effects of FA-DOX-BSA MNPs in vitro \\ MTT}

For this study, KB cells were heated to a defined temperature $\left(43^{\circ} \mathrm{C}\right)$ on the heating coil of a high-frequency induction heater ( $\mathrm{f}=200 \mathrm{kHz} ; \mathrm{I}=20 \mathrm{~A}$ ) for 60 minutes. The antitumor effect on KB cells was determined by measuring the cell IR by MTT assay after 48 hours of drug treatment. As depicted in Table 1, the cell IRs for the DOX-BSA NP and DOX-BSA MNP groups were $22.50 \%$ and $25.20 \%$, respectively. After using FA-DOX-BSA MNPs containing the same drug concentration, the cell IR increased to $43.15 \%$. Thus, FA-DOX-BSA MNPs was effective in terms of inhibiting KB cell growth $(P<0.05)$. Additionally, placement of the BSA MNP, DOX-BSA MNP, and FA-DOX-BSA MNP groups in an alternating magnetic field ( $\mathrm{f}=200 \mathrm{kHz} ; \mathrm{I}=20 \mathrm{~A}$ ) for one hour of heating resulted in an increase in cell IRs to $47.84 \%, 74.06 \%$, and $97.79 \%$, respectively, indicating that heating itself inhibits KB cell growth. Cell viability in the negative control group, the negative control group with heating, and the BSA MNP group with no heating was not affected. These results suggest that FA-DOX-BSA MNPs have enhanced antitumor efficacy. When combined with hyperthermia, FA-DOX-BSA MNPs were more effective in terms of inhibiting cell proliferation than the other groups.

\section{Apoptosis assay}

The number of apoptotic KB cells after 48 hours of incubation is shown in Figure 6. Apoptosis and necrosis can be found in all treatment groups, and the apoptosis take up a big proportion. It showed that the main function of our therapy was to induce apoptosis of $\mathrm{KB}$ cells in vitro. The function of hyperthermia at temperatures ranging from $42^{\circ} \mathrm{C}$ to $45^{\circ} \mathrm{C}$ is to cause inhibition of cellular enzymes and cell damage largely through the process of apoptosis. ${ }^{29,30}$ Using our therapy, the magnetic nanoparticle hyperthermia groups induced apoptosis even in the absence of doxorubicin. Induction of apoptosis after 

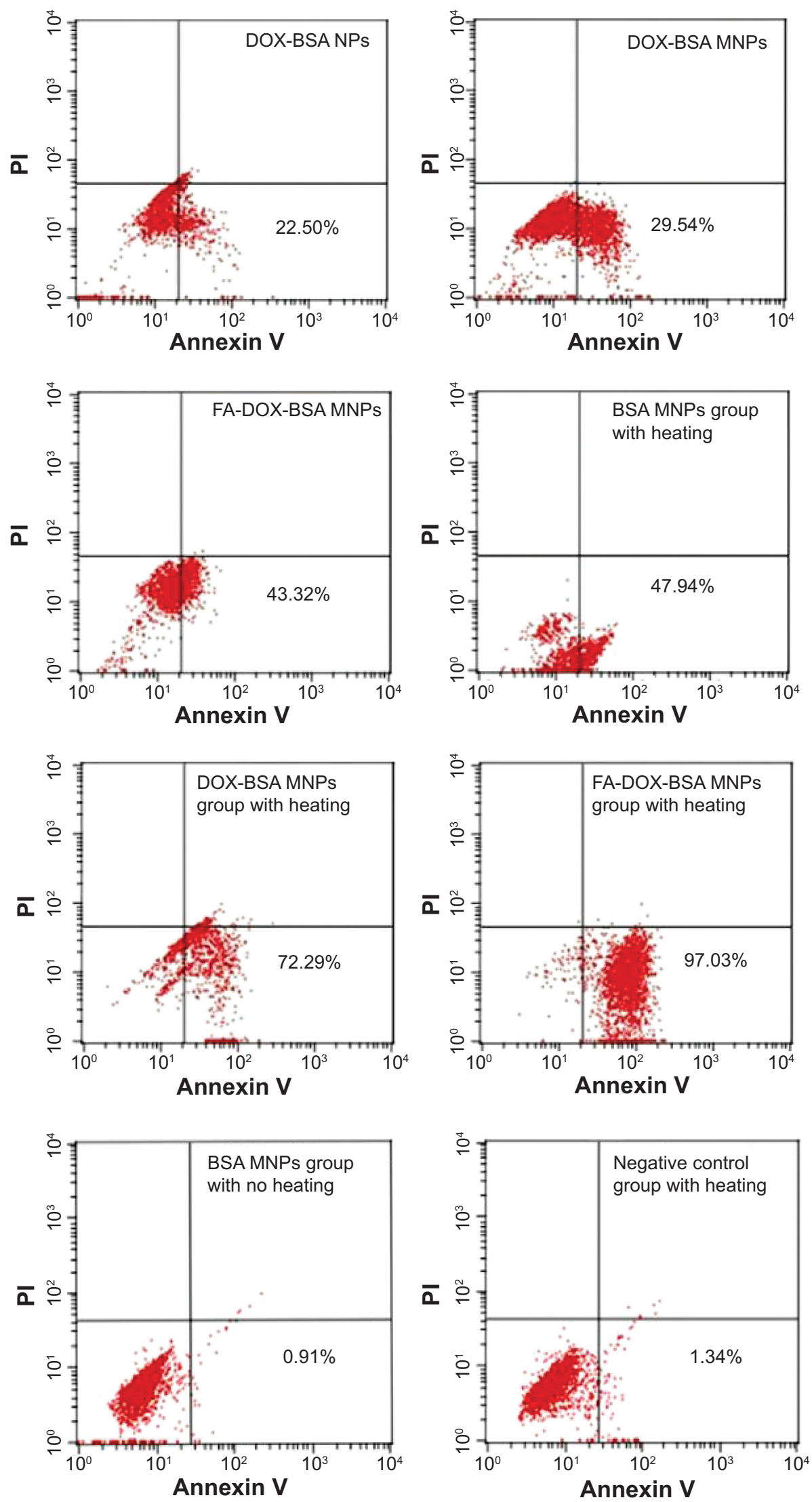

Figure 6 Apoptotic cell contents as determined by flow cytometry.

Notes: Apoptosis existed in every treatment group. Fluorescence percentage is included in the flow cytometer scatter diagrams.

Abbreviations: FA, folic acid; DOX, doxorubicin; BSA, bovine serum albumin; MNPs, magnetic nanoparticles; NPs, nanoparticles; PI, propidium iodide. 
Table I Inhibition rate for nanoparticles against nasopharyngeal carcinoma KB cells

\begin{tabular}{lll}
\hline Group & Optical density (mean $\pm \mathbf{S D}, \mathbf{n}=\mathbf{6})$ & Inhibition rate (\%) \\
\hline a) Negative control & $1.087 \pm 0.01 I^{\mathrm{a}, \mathrm{b}, \mathrm{c}}$ & 0 \\
b) DOX-BSA NP & $0.842 \pm 0.01 I^{\mathrm{a}}$ & $22.50 \pm 1.2$ \\
c) DOX-BSA MNP & $0.813 \pm 0.010^{\mathrm{a}}$ & $25.20 \pm 1.0$ \\
d) FA-DOX-BSA MNP & $0.618 \pm 0.019^{\mathrm{a}, \mathrm{b}}$ & $43.15 \pm 2.1$ \\
e) BSA MNP with heating & $0.567 \pm\left. 0.02\right|^{\mathrm{a}, \mathrm{b}}$ & $47.84 \pm 0.99$ \\
f) DOX-BSA MNP with heating & $0.282 \pm 0.012^{\mathrm{a}, \mathrm{b}}$ & $74.06 \pm 3.01$ \\
g) FA-DOX-BSA MNP with heating & $0.024 \pm 0.01 I^{\mathrm{a}, \mathrm{b}}$ & $97.79 \pm 1.45$ \\
h) Negative control with heating & $1.083 \pm 0.022^{\mathrm{c}}$ & $0.37 \pm 0.08$ \\
i) BSA MNP with no heating & $1.081 \pm 0.015^{\mathrm{c}}$ & $0.55 \pm 0.02$ \\
\hline
\end{tabular}

Notes: a $P<0.05$, comparison of experimental groups with the negative control group; ${ }^{b P}<0.05$, comparison of group (g) with group (d), (e), and (f); ${ }^{c P>0.05}$, comparison of groups (h) and (i) with negative control group.

Abbreviations: DOX, doxorubicin; BSA, bovine serum albumin; NPs, nanospheres; MNPs, magnetic iron oxide nanospheres; SD, standard deviation; FA, folic acid.

treatment with doxorubicin magnetic nanoparticles combined with hyperthermia was considerably greater than in the doxorubicin nanoparticle and magnetic nanoparticle hyperthermia groups $(P<0.05)$. This means that hyperthermia had a positive impact on the effect of chemotherapy. Additionally, FA-DOX MNPs combined with hyperthermia induced apoptosis more rapidly than did nontargeted magnetic nanoparticles at an identical doxorubicin concentration $(P<0.05)$. Therefore, a combined therapy mode could achieve a higher rate of apoptosis in NPC KB cells than chemotherapy alone. The percentages of apoptotic cells after treatment with DOX-BSA NPs, DOX-BSA MNPs, FA-DOX-BSA MNPs, BSA MNPs with heating, DOX-BSA MNPs with heating, FA-DOXBSA MNPs with heating, the negative control with heating, and BSA MNPs with no heating were $23.68 \% \pm 3.01 \%$, $26.24 \% \pm 2.94 \%, 43.87 \% \pm 3.92 \%, 48.33 \% \pm 2.01 \%$, $77.32 \% \pm 6.67 \%, 96.24 \% \pm 2.81 \%, 0.84 \% \pm 2.1 \%$ and $1.01 \% \pm 0.25 \%$, respectively (Table 2 ). These findings are consistent with the MTT data shown in Table 1.

Table 2 Apoptotic cell contents as determined by flow cytometry

\begin{tabular}{ll}
\hline Group & $\begin{array}{l}\text { Apoptotic cells (\%) } \\
(\mathbf{m e a n} \pm \mathbf{S D}, \mathbf{n}=\mathbf{6})\end{array}$ \\
\hline a) Negative control & $0^{\mathrm{a}, \mathrm{c}}$ \\
b) DOX-BSA NP & $23.68 \pm 3.0 \mathrm{I}^{\mathrm{a}}$ \\
c) DOX-BSA MNP & $26.24 \pm 2.94^{\mathrm{a}}$ \\
d) FA-DOX-BSA MNP & $43.87 \pm 3.92^{\mathrm{a}, \mathrm{b}}$ \\
e) BSA MNP with heating & $48.33 \pm 2.0 \mathrm{I}^{\mathrm{a}, \mathrm{b}}$ \\
f) DOX-BSA MNP with heating & $77.32 \pm 6.67^{\mathrm{a}, \mathrm{b}}$ \\
g) FA-DOX-BSA MNP with heating & $96.24 \pm 2.8 \mathrm{I}^{\mathrm{a}, \mathrm{b}}$ \\
h) Negative control with heating & $0.84 \pm 2.1^{\mathrm{c}}$ \\
i) BSA MNP with no heating & $1.01 \pm 0.25^{\mathrm{c}}$ \\
\hline
\end{tabular}

Notes: a ${ }^{C}$ omparison of experimental groups with negative control group, $P<0.05$; ${ }^{b}$ Comparison of group (g) with group (d), (e), and (f) $P<0.05 ; \mathrm{c} P>0.05$, comparison of groups (h) and (i) with negative control group.

Abbreviations: DOX, doxorubicin; BSA, bovine serum albumin; NPs, nanospheres; MNPs, magnetic iron oxide nanospheres; SD, standard deviation; FA, folic acid.

\section{Antitumor effect of FA-DOX-BSA MNPs in vivo}

We determined the effect of FA-DOX-BSA MNPs on NPC tumor growth by calculating the changes in tumor volume and weight and by hematoxylin-eosin staining. During this process, the temperature inside a tumor can reach $41^{\circ} \mathrm{C}-44^{\circ} \mathrm{C}$. Hemorrhage, necrosis, and eschar were evident throughout treatment, and hyperthermia-treated and thermochemotherapy-treated gray-white tumor tissue showed a dark brown color at the end of the treatment cycle. No such finding was evident in the control and chemotherapy only groups. Tumor volume and weight IRs are shown in Table 3. Consistent with our previous in vitro findings, FA-DOX-BSA MNPs combined with hyperthermia yielded significantly higher inhibition rates than the other groups $(P<0.05)$. Specifically, the folic acid-targeted and nonheated groups exhibited decreased tumor volume and weight, respectively, from $0 \%$ with each pretreatment to $83.0 \%$ and $60.6 \%$, respectively, at 1 week post-treatment. By contrast, the heat-treated, folic acid-targeted groups showed inhibition rates of $97.1 \%$ and $77.32 \%$, respectively, over the same period. Hematoxylin-eosin staining revealed that tumor cells proliferated actively in the control group, with a low degree of differentiation (Figure 7). However, in the thermal therapy groups, some tumor tissues showed obvious necrosis and hemorrhage, particularly in the folic acid-targeted groups, where the tumor structures were completely destroyed and the tumor cells were irregularly shaped. The most intense inflammatory reaction and infiltration by inflammatory cells were also found in the thermal therapy groups. Brown iron was deposited in the tumors in the heated groups. These findings suggested that folic acidtargeted hyperthermia combined with chemotherapy could kill tumor cells. Taken together, these data demonstrate that administration of FA-DOX-BSA MNP is an effective 
Table 3 Inhibition rate of tumor volume and tumor weight

\begin{tabular}{lllll}
\hline Group & $\begin{array}{l}\text { Tumor volume }\left(\mathbf{m m}^{3}\right), \\
(\mathbf{m e a n} \pm \mathbf{S D}, \mathbf{n}=\mathbf{6})\end{array}$ & $\begin{array}{l}\text { Tumor mass } \mathbf{( g )} \\
(\mathbf{m e a n} \pm \mathbf{S D}, \mathbf{n = 6})\end{array}$ & IR of volume (\%) & IR of weight (\%) \\
\hline a) Negative control & $0.524 \pm 0.03^{\mathrm{a}, \mathrm{b}}$ & $0.657 \pm 0.038^{\mathrm{a}}$ & 0 & 0 \\
b) DOX-BSA NP & $0.276 \pm 0.098^{\mathrm{a}}$ & $0.547 \pm 0.058^{\mathrm{a}}$ & 47.4 & 16.7 \\
c) DOX-BSA MNP & $0.312 \pm 0.224^{\mathrm{a}}$ & $0.549 \pm 0.045^{\mathrm{a}}$ & 40.4 & 16.4 \\
d) FA-DOX-BSA MNP & $0.089 \pm 0.046^{\mathrm{a}}$ & $0.259 \pm 0.057^{\mathrm{a}, \mathrm{b}}$ & 83.0 & 60.6 \\
e) BSA MNP with heating & $0.132 \pm 0.039^{\mathrm{a}}$ & $0.462 \pm\left. 0.08\right|^{\mathrm{a}}$ & 74.8 & 29.7 \\
f) DOX-BSA MNP with heating & $0.049 \pm 0.015^{\mathrm{a}}$ & $0.362 \pm 0.073^{\mathrm{a}, \mathrm{b}}$ & 90.6 & 44.9 \\
g) FA-DOX-BSA MNP with heating & $0.015 \pm 0.006^{\mathrm{a}, \mathrm{b}}$ & $0.151 \pm 0.038^{\mathrm{a}, \mathrm{b}}$ & 97.1 & 77.0 \\
\hline
\end{tabular}

Notes: a Comparison of experimental groups with negative control group, $P<0.05$; bcomparison of FA-DOX-BSA MNP group that was heated under an alternating magnetic field with the other groups, $P<0.05$.

Abbreviations: IR, inhibition rate; DOX, doxorubicin; BSA, bovine serum albumin; NPs, nanospheres; MNPs, magnetic iron oxide nanospheres; FA, folic acid; SD, standard deviation.

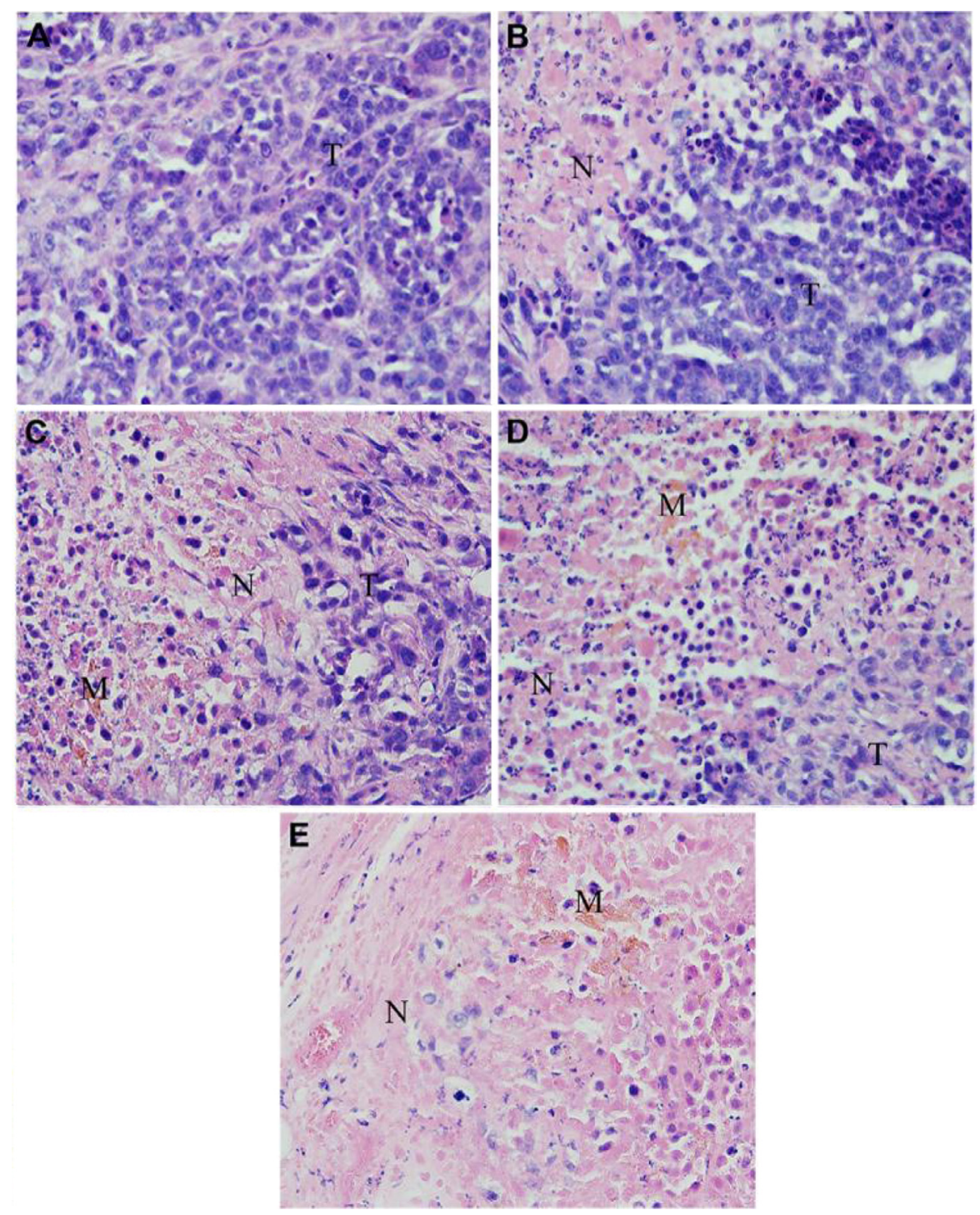

Figure 7 Histopathological changes in NPC xenograft tumors (stained with hematoxylin-eosin, 40x).

Notes: (A) Negative control group. (B) DOX-BSA NP group. Inflammation can be observed in the tissues. (C) BSA MNP group with heating. A large amount of inflammatory cell infiltration and some spotty necrosis were noted. Brown magnetic materials were deposited in the tissue. (D) DOX-BSA MNP group with heating. Similar to (C), brown magnetic materials, inflammatory cell infiltration, and spotty necrosis are seen in the tissue and cells, albeit to a greater degree. (E) FA-DOX-BSA MNP group with heating. The effect in this group was the most different compared with the other groups. Massive necrosis with marked cell disintegration is evident throughout the image.

Abbreviations: T, tumor tissue; N, necrosis; M, materials of $\mathrm{Fe}_{3} \mathrm{O}_{4}$; FA, folic acid; DOX, doxorubicin; BSA, bovine serum albumin; MNP, magnetic nanoparticles; NP, nanoparticles; NPC, nasopharyngeal carcinoma. 
method for tumor chemotherapy. Heating combined with chemotherapy had the most significant effect on tumors.

FA-DOX-BSA MNPs combined with hyperthermia therapy had a significantly greater effect on average tumor volume and weight than either non-FA-DOX-BSA MNPs or chemotherapy alone. In contrast, histopathological evaluation indicated that tumor structures in the FA-DOX-BSA MNP group were destroyed more completely than those in the non-FA-DOX-BSA MNP group. It is likely that the following mechanisms are involved:

1. Hyperthermia as an effective treatment modality to augment chemotherapy-based anticancer treatments. Hyperthermia in combination chemotherapy can not only increase the drug concentration in tumor cells but also reduce the toxic effects to normal tissue and prevent the development of drug resistance. Clinical tests have shown that hyperthermia combined with chemotherapy has increased efficacy against tumors such as high-grade soft tissue sarcomas. ${ }^{31}$ In our experiment, when combined with hyperthermia therapy, FA-DOX-BSA MNPs had a greater effect on cell viability and inhibited NPC cells significantly (a four-fold increase in cytotoxicity versus DOX-BSA MNPs).

2. $\mathrm{Fe}_{3} \mathrm{O}_{4}$ has a short half-life and no specific tumor-targeting effect, which limits its application in tumor therapy. In our study, we incorporated $\mathrm{Fe}_{3} \mathrm{O}_{4}$ into BSA NPs to avoid them being taken up by reticuloendothelial cells and removed by macrophages. BSA NPs with a smaller size (50-300 nm) compared with microparticles are easily prepared and could be widely accepted in the pharmaceutical industry.

3. Tumor cells have a unique ability to absorb magnetic nanoparticles (8-400-fold more than normal cells) and distribute them in daughter cells uniformly, making them highly susceptible to magnetic fluid hyperthermia and avoiding damage to normal tissues. ${ }^{32}$

4. FA-DOX-BSA MNPs showed a two-fold increase in cytotoxicity compared with nontargeted nanoparticles.

Active targeting of folic acid is a promising approach via which a drug delivery system can reach and penetrate into tumor cells with overexpression of folate receptors on their surface, and then release its encapsulated therapeutic agent in a controlled and sustained manner. FA-BSA MNPs had less in vitro toxicity and higher antitumor activity. Inhibition of in vivo tumor growth demonstrated that these carriers have excellent antitumor activity and induce a high rate of apoptosis in cancer cells.

\section{Conclusion}

Several recent studies have investigated the synthesis of folic acid-targeted albumin nanoparticles to avoid the side effects of antitumor drugs and improve tumor-targeted drug delivery to improve the treatment of tumors. However, FA-DOX-BSA MNPs targeting NPC are rarely used. In this study, we successfully synthesized FA-DOX-BSA MNPs as nanocarriers, and achieved thermotherapy, chemotherapy, and targeted therapeutic effects against NPC. However, further studies must be performed to optimize this therapy for clinical use and determine whether it can be used to treat other tumors.

\section{Acknowledgments}

This work was supported by grants from the National Key Basic Research Program of China (973 Program; 2013 CB933904, 2011CB933500) and the National Natural Science Foundation of China (81271635, 81071881, 81301270, 81101139).

\section{Disclosure}

The authors report no conflicts of interest in this work.

\section{References}

1. Glastonbury CM, Salzman KL. Pitfalls in the staging of cancer of NPC. Neuroimaging Clin N Am. 2013;23:9-25.

2. Han BL, Xu XY, Zhang CZ, et al. Systematic review on Epstein-Barr virus (EBV) DNA in diagnosis of NPC in Asian populations. Asian Pac J Cancer Prev. 2012;13:2577-2581.

3. Agudelo D, Bourassa P, Bruneau J, Bérubé G, Asselin E, Tajmir-Riahi HA. Probing the binding sites of antibiotic drugs DOX and N-(trifluoroacetyl) DOX with human and BSAs. PLoS One. 2012; 7:e43814.

4. Hanušová V, Boušová I, Skálová L. Possibilities to increase the effectiveness of DOX in cancer cells killing. Drug Metab Rev. 2011;43: 540-557.

5. Carvalho C, Santos RX, Cardoso S, et al. DOX: the good, the bad and the ugly effect. Curr Med Chem. 2009;16:3267-3285.

6. Kratz F. Albumin as a drug carrier: design of prodrugs, drug conjugates and nanoparticles. J Control Release. 2008;132:171-183.

7. Zhao D, Zhao X, Zu Y, et al. Preparation, characterization, and in vitro targeted delivery of folic-decorated paclitaxel-loaded BSA nanoparticles. Int J Nanomedicine. 2010;5:669-677.

8. Saxena V, Naguib Y, Hussain MD. Folic receptor targeted 17allylamino-17-demethoxygeldanamycin (17-AAG) loaded polymeric nanoparticles for breast cancer. Colloids Surf B Biointerfaces. 2012;94: 274-280.

9. Lee KY, Seow E, Zhang Y, Lim YC. Targeting CCL21-folic acidupconversion nanoparticles conjugates to folic receptor- $\alpha$ expressing tumor cells in an endothelial-tumor cell bilayer model. Biomaterials. 2013;34:4860-4871.

10. Rijnboutt S, Jansen G, Posthuma G, Hynes JB, Schornagel JH, Strous GJ. Endocytosis of GPI-linked membrane folate receptor-alpha. J Cell Biol. 1996;132:35-47.

11. Birn H, Selhub J, Christensen EI. Internalization and intracellular transport of folate-binding protein in rat kidney proximal tubule. $\mathrm{Am} \mathrm{J}$ Physiol. 1993;264:C302-C310. 
12. Hall EJ, Cox JD. Physical and biological basis of radiation therapy. In: Moss WT, Brand WN, Battifora H, editors. Radiation Oncology: Rationale, Technique, Results. St Louis, MO, USA: CV Mosby Co; 2003.

13. Pelicci PG, Dalton P, Orecchia R. Heating cancer stem cells to reduce tumor relapse. Breast Cancer Res. 2011;13:305.

14. Gilchrist RK, Medal R, Shorey WD, Hanselman RC, Parrott JC, Taylor CB. Selective inductive heating of lymph nodes. Ann Surg. 1957;146:596-606.

15. Wang L, Zhang J, An Y, et al. A study on the thermochemotherapy effect of nanosized As2O3/MZF thermosensitive magnetoliposomes on experimental hepatoma in vitro and in vivo. Nanotechnology. 2011; 22:315102.

16. Hayashi K, Nakamura M, Sakamoto W, et al. Superparamagnetic nanoparticle clusters for cancer theranostics combining magnetic resonance imaging and hyperthermia treatment. Theranostics. 2013;3:366-376.

17. Kumar M, Singh G, Arora V, et al. Cellular interaction of folate acid conjugated superparamagnetic iron oxide nanoparticles and its use as contrast agent for targeted magnetic imaging of tumor cells. Int $J$ Nanomedicine. 2012;7:3503-3516.

18. Jordan A, Scholz R, Wust P, et al. Effects of magnetic fluid hyperthermia (MFH) on C3H mammary carcinoma in vivo. Int J Hyperthermia. 1997; 13:587-605.

19. Li XH, Rong PF, Jin HK, Wang W, Tang JT. Magnetic fluid hyperthermia induced by radiofrequency capacitive field for the treatment of transplanted subcutaneous tumors in rats. Exp Ther Med. 2012;3: 279-284.

20. Béalle G, Di Corato R, Kolosnjaj-Tabi J, et al. Ultra magnetic liposomes for MR imaging, targeting, and hyperthermia. Langmuir. 2012;28: 11834-11842.

21. Tang QS, An YL, Yang R. In vitro and in vivo evaluation of tumortargeting efficacy of folate-conjugated magnetic albumin nanospheres by MRI. Chinese Journal of Magnetic Resonance. 2013;30:499-506.

22. Li Y, Liu J, Zhong Y, et al. Biocompatibility of $\mathrm{Fe}_{3} \mathrm{O}_{4} @$ Au composite magnetic nanoparticles in vitro and in vivo. Int J Nanomedicine. 2011;6 2805-2819.
23. Chen D, Tang Q, Li X, et al. Biocompatibility of magnetic $\mathrm{Fe}_{3} \mathrm{O}_{4}$ nanoparticles and their cytotoxic effect on MCF-7 cells. Int J Nanomedicine. 2012;7:4973-4982.

24. Foster JF. In: Rosenoer VM, Oratzand M, Rothschild MA, editors Albumin Structure, Function and Uses. Oxford: Pergamon Press; 1977:53-84.

25. Foster JF. In: Putnam FW, editor. The Plasma Proteins. 1st ed. Vol. 1. New York: Academic Press; 1960:179-239.

26. Budavari S, O’Neil MJ, Smith A, Heckleman PE, Kinneary JF, editors. The Merck Index: An Encyclopedia of Chemicals, Drugs, and Biologicals. Whitehouse Station, NJ, USA: Merck; 1989.

27. Ak G, Yilmaz H, Sanlıer SH. Preparation of magnetically responsive albumin nanospheres and in vitro drug release studies. Artif Cells Nanomed Biotechnol. 2014;42:18-26.

28. Shen Z, Li Y, Kohama K, et al. Improved drug targeting of cancer cells by utilizing actively targetable folic acid-conjugated albumin nanospheres. Pharmacol Res. 2011;63:51-58.

29. Overgaard J, Suit HD. Time-temperature relationship in hyperthermic treatment of malignant and normal tissue in vivo. Cancer Res. 1979;39: 3248-3253.

30. Tao Y, Guo Y, Liu W, et al. AKT inhibitor suppresses hyperthermiainduced Ndrg2 phosphorylation in gastric cancer cells. Braz J Med Biol Res. 2013;46:394-404.

31. Franckena M, De Wit R, Ansink AC, et al. Weekly systemic cisplatin plus locoregional hyperthermia: an effective treatment for patients with recurrent cervical carcinoma in a previously irradiated area. Int $J$ Hyperthermia. 2007;23:443-450.

32. Jordan A, Wust P, Scholz R, et al. Cellular uptake of magnetic fluid particles and their effects on human adenocarcinoma cells exposed to AC magnetic fields in vitro. Int J Hyperthermia. 1996;12:705-722.
International Journal of Nanomedicine

\section{Publish your work in this journal}

The International Journal of Nanomedicine is an international, peerreviewed journal focusing on the application of nanotechnology in diagnostics, therapeutics, and drug delivery systems throughout the biomedical field. This journal is indexed on PubMed Central, MedLine, CAS, SciSearch $®$, Current Contents $\AA /$ Clinical Medicine,

\section{Dovepress}

Journal Citation Reports/Science Edition, EMBase, Scopus and the Elsevier Bibliographic databases. The manuscript management system is completely online and includes a very quick and fair peer-review system, which is all easy to use. Visit http://www.dovepress.com/ testimonials.php to read real quotes from published authors. 